Voix et Images

\title{
Gaston Miron ou la contradiction illuminante - Jacques Rancourt - Hélène Dorion
}

\section{André Brochu}

Volume 36, numéro 2 (107), hiver 2011

URI : https://id.erudit.org/iderudit/1002452ar

Aller au sommaire du numéro

Éditeur(s)

Université du Québec à Montréal

ISSN

0318-9201 (imprimé)

1705-933X (numérique)

Découvrir la revue

Citer ce compte rendu

Brochu, A. (2011). Compte rendu de [Gaston Miron ou la contradiction illuminante - Jacques Rancourt - Hélène Dorion]. Voix et Images, 36(2), $160-165$. d'utilisation que vous pouvez consulter en ligne.

https://apropos.erudit.org/fr/usagers/politique-dutilisation/ 


\section{P O ÉS I E}

Gaston Miron ou la contradiction illuminante Jacques Rancourt - Hélène Dorion

$+++$

\section{ANDRÉ BROCHU}

Université de Montréal

Au moment de rendre compte du recueil d'entretiens de Gaston Miron, L'avenir dégage $^{1}$, je suis frappé par le nombre de liens que le poète entretenait avec une institution importante mais souvent éclipsée dans le public par des maisons d'enseignement plus jeunes (ou plus dynamiques ?) : l'Université de Montréal. C'est la revue Études françaises, en 1970, qui sous l'impulsion de son directeur, Georges-André Vachon, et de Jacques Brault décerne son prix à Gaston Miron et publie du même coup son œuvre maîtresse, L'homme rapaillé. Mais auparavant, Gilles Marcotte avait fait paraître des articles sur l'Hexagone et sur son principal fondateur, et Jacques Brault avait consacré une étude remarquable à Miron le magnifique. Mentionnons aussi Lise Gauvin, qui fut la collaboratrice de Miron pour certains ouvrages et qui, en 1999, alors directrice de la revue Études françaises, a préparé un numéro double intitulé Gaston Miron : un poète dans la cité (elle y souligne d'ailleurs avant moi les nombreux liens du poète avec le Département d'études françaises - aujourd'hui Département des littératures de langue française). Citons encore Pierre Popovic et Jean Larose qui ont publié soit des études sur lui, soit de substantiels entretiens. Pierre Nepveu, surtout, a réalisé sur Miron différents travaux (avec Marie-Andrée Beaudet, professeure à l'Université Laval, qui fut la compagne du poète), et prépare depuis plusieurs années une biographie monumentale et très attendue sur l'auteur de L'homme rapaillé. Ajoutons à cela que Gaston Miron fut plusieurs fois invité à rencontrer les étudiants de l'université, qu'il y fut écrivain en résidence, et rappelons que, lors de la crise d'Octobre de 1970, un colloque tenu à l'Université de Montréal lui fut consacré pour dénoncer son emprisonnement. Et j'en passe... Je veux seulement souligner ici la proximité de l'écrivain avec une institution sérieuse et pas particulièrement remuante, proximité qui éclaire une facette de sa personnalité. Issu d'un milieu sylvicole et populaire (Sainte-Agathe-des-Monts), étranger à la petitebourgeoisie qui forme la clientèle courante de l'université, Gaston Miron a su s'imposer, sans rien renier de ses origines, comme un homme de savoir aussi bien que

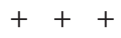

1 Gaston Miron, L'avenir dégagé. Entretiens 1959-1993, édition préparée par Marie-Andrée Beaudet et Pierre Nepveu, Montréal, l’Hexagone, 2010, 432 p. 
comme l'éclatant poète qu'il est, aux pieds solidement ancrés dans le terreau douloureux et espérant de son pays.

Évidemment, les contributions variées de professeurs de l'Université de Montréal constituent une part seulement, parmi d'autres, de tout ce qui a permis la connaissance et la reconnaissance du poète, mais cette part compte sûrement au nombre des plus représentatives. Il convenait de la souligner.

Miron le poète inspiré, Miron l'homme de culture: sans doute. Mais il est aussi, on le sait, Miron le militant, infatigable partisan de l'indépendance du Québec, bien qu'il sût faire la part des choses et éviter les confusions entre les différentes tâches qu'il devait assumer. Pour lui, la poésie ne doit pas être platement politique, même s'il est donné à celle-ci d'exprimer les plus hauts espoirs d'une collectivité. Cette expression du pays n'est en rien monosémique, elle transite par la totalité des sens que véhicule le poème. Elle est tout de même indispensable à la nature même de l'œuvre. On peut dire de cette dernière qu'elle lie en un faisceau unique des réalités très diverses, ce qui fait sa richesse et l'impossibilité pour son auteur de séparer les diverses tonalités constitutives de son inspiration. Voilà pourquoi il y a UN livre de Miron. Il ne peut en être autrement. Il y a L'homme rapaillé, fait du rapaillage de plusieurs œuvres nées séparément mais qui ne trouvent d'accomplissement que dans leur réunion en un seul livre. Les Poèmes épars ${ }^{2}$, complément sans doute indispensable, souffriront toujours de leur absence d'intégration au seul grand livre. Et les entretiens ici réunis par Pierre Nepveu et Marie-Andrée Beaudet, d'une certaine façon, malgré la diversité des interlocuteurs de Miron, de Michel Roy à Lise Gauvin en passant par Jean Royer, Raoul Roy, Claude Filteau, Jean Larose, Flávio Aguiar et plusieurs autres, constituent un seul entretien sans cesse repris, refait, poussé plus loin, avec des redites considérables et un projet énorme: dire la contradiction.

«J'ai toujours été dans la contradiction et le paradoxe», affirme Gaston Miron, interrogé sur sa relation complexe avec la tradition poétique «canadienne-française » (Englebert Gallèze) et la modernité (254). Le poète milite pour une poésie nationale et non nationaliste, pour une inspiration sociale et non socialiste, mais cela ne va pas sans quelques pas de danse (de gigue!) sur la corde raide.

La contradiction, sujet central de son œuvre et de sa vie, est avant tout la périlleuse conciliation de l'un et du multiple, et rejoint le scandale d'un livre - L'homme rapaillé - qui est en même temps tous les livres de l'auteur. Ou celle d'un entretien qui veut chaque fois dire la totalité de l'être et des choses, du poème et du monde:

C'est toujours la démarche que j'ai eue, j'ai essayé d'avoir une démarche totalisante, confie-t-il au journaliste Pierre Paquette. On ne peut jamais être un homme totalisé, mais on peut essayer d'avoir une démarche qui englobe le plus de choses possibles.

$$
+++
$$

2 Gaston Miron, Poèmes épars, édition préparée par Marie-Andrée Beaudet et Pierre Nepveu, Montréal, l’Hexagone, coll. «L'appel des mots», 2003, 134 p. 
[...] Mais, au cœur de tout, il y avait cette idée centrale de création, de beauté...

(162-163)

Dans un poème, évoquer le foisonnement du monde n'est possible que si l'entreprise est, en son cœur même, esthétique. C'est l'acte créateur de beauté qui permet de convoquer des éléments pris aux sources les plus hétérogènes, la nature, le drame humain, l'amour, la nation, la société ; d'opérer la fulgurante fusion des contraires, de se mesurer au paradoxe strident de l'existence. La beauté transmute en vérité universelle l'encombrement du moi immédiat. Quoi de plus particulier qu'un énoncé comme : «j'ai du chiendent d'achigan dans l'âme» (374)? Gaston Miron commente : «Le chiendent, c'est difficile à arracher. L'achigan est à l'eau ce que le chiendent est à la terre : ça ne sort pas. » (374) La plante et le poisson représentent ce qu'il y a de mauvais, sur la terre et dans l'eau, et symbolisent l'un et l'autre, l'un par l'autre, l'insubordination du poète dans la société. La force brouillonne de l'image, si éloignée des métaphores usuelles, est saisissante et crée l'universel.

Je dis «brouillonne», car on pourrait reprocher à "chiendent d'achigan» une mise en relation brutale de deux réalités trop éloignées. Gaston Miron est très conscient de ces coups de force sémantiques qui font la beauté et la complexité de nombre de ses images. Il les appelle parfois «incongruités» (372) et peut passer plusieurs années à tenter de les réduire, ce qui explique l'instabilité de ses textes et l'absence proclamée, pour L'homme rapaillé, de version définitive. Le livre unique ne cesse de bouger, de s'affirmer dans la pluralité de ses variantes. Certes, il s'agit toujours d'approcher davantage de la beauté, sans rien sacrifier de son arrière-plan signifiant, mais la beauté n'est vraiment atteinte que si la raison raisonnante se trouve respectée. Ce travail rejoint celui, non plus linguistique mais idéologique, de la désaliénation que Gaston Miron dit pratiquer sur son poème : « Je suis toujours sur le terrain d'un poème, ça peut durer dix ans. Parce que chaque fois que je découvre une aliénation à mon poème, je désaliène mon poème et, par le fait même, je me désaliène moi-même.» (258) On voit quelle exigence presque surhumaine préside, chez Miron, à la création simultanée du poème et de soi.

L'avenir «dégagé»? Qu'il parle de son enfance, de son peuple aliéné, de l'indispensable libération collective, de son œuvre toujours à faire, de la langue, Gaston Miron projette partout l'image d'un homme généreux, d'une intelligence prodigieuse - celle de l'intellectuel primaire, au bon sens du terme, qui était aussi l'intelligence incomparable d'un Julien Bigras - , et d'un grand créateur, à la fois humble et ayant l'orgueil de ses ambitions. L'avenir dégagé annonce, dans le poème intitulé «L'oc tobre $^{3}$ ", «l'avenir engagé ", c'est-à-dire une sorte de grand soir où l'homme réaliserait ses aspirations personnelles et collectives. L'avenir dégagé, dont nous parle surtout Gaston Miron à travers les différents sujets qu'il aborde, reste en deçà de ce paradis sur terre et il est conforme à la vérité d'un poète dont tout l'espoir, qui est aussi le nôtre, s'est fait langage et promesse de vie.

3 Gaston Miron, L'homme rapaillé, texte annoté par l'auteur, préface de Pierre Nepveu, Montréal, l'Hexagone, 1994, p. 88. 
Jacques Rancourt publie un deuxième choix de poèmes ${ }^{4}$, préfacé par le poète, théoricien et polémiste français Henri Meschonnic. Le choix précédent, Les choses sensibles ${ }^{5}$, couvrait les quinze premières années de production. Vingt années se sont ajoutées depuis.

On pourrait penser que ces deux livres, qui retiennent le meilleur de la production de l'auteur, sont en bonne partie conçus pour le public québécois, les recueils dont leur matière est extraite ayant été édités à l'étranger. Jacques Rancourt, né au Québec et attaché à son pays, n'en a pas moins vécu presque toute sa vie adulte en France. Il s'est d'abord consacré à des études universitaires qu'il a poursuivies jusqu'au doctorat, puis à diverses activités littéraires, souvent en collaboration avec des écrivains français ou européens. L'une de ces activités est la traduction, et Jacques Rancourt est, à Paris, l'un des littéraires qui s'est le plus dévoué au rapprochement des domaines anglais et français. Voilà une façon très «canadienne» de vivre l'éloignement (peut-on parler d'exil?).

Devant la poésie de Jacques Rancourt, on n'a pas vraiment l'impression d'être en face d'une inspiration québécoise, sauf dans de rares poèmes à saveur quelque peu folklorique - l'un, notamment, qui fait revivre Gaston Miron décédé depuis peu:

\footnotetext{
Vas-y Gaston j'entends l'harmonica

j'entends des pieds qui battent

et qui marquent la mesure (144).
}

Le poète partage avec plusieurs cousins hexagonaux la fascination pour ce diable d'homme «rapaillé» qui a su incarner si bien les espérances de son peuple.

Pas tout à fait québécois donc dans son inspiration, Rancourt l'est pourtant dans plusieurs des traits qui caractérisent sa poésie. Signalons d'abord sa simplicité, puis son côté discrètement enjoué, lesquels rendent sa lecture fort agréable. Le premier recueil, La journée est bien partie pour durer, nous installe dès le titre dans une tonalité existentielle qui a quelque chose d'immédiat, de quotidien, en même temps que d'un peu étrange. Les choses, le réel sont à portée de main, mais prêts aux métamorphoses. Car il arrive que les lois du monde se compliquent, et le réalisme tourne alors au doux surréalisme. La vie de couple, qui est au cœur du quotidien, s'en trouve presque ébranlée:

on peut être deux et s'émietter

je peux dormir à l'ombre comme un veau

je peux aussi te prendre comme une valise

tu coules à pic et tu t'accroches aux herbes et tu caches

ta tête sous les plantes

4 Jacques Rancourt, Veilleur sans sommeil. Choix de poèmes 1974-2008, Montréal/Pantin, Éditions du Noroît/Le Temps des cerises, coll. «Ovale», 2010, 208 p. 5 Jacques Rancourt, Les choses sensibles. Choix de poèmes 19741988, Montréal, l'Hexagone, 1991, 104 p. 
je te fais parfois l'amour comme un café sans sucre

et je ne suis pas drôle (25).

Pas drôle... à moins qu'on ne soit attentif aux jeux de langage, par exemple à cette façon de détourner l'expression québécoise prendre pour une valise en cette autre, prendre comme une valise, qui est absurde ou, peut-être, un peu salace...

Rarement porté vers les thèmes nationalistes, Rancourt ne fait guère plus de place aux réalités sociales ( «Il ne suffira pas de quelques mots velus/pour loger les dormeurs du métro/pour abriter les vagabonds des villes » [117]) ou aux catastrophes et calamités mondiales ( «nous sommes aux deux tiers du printemps/avec des nuages sur les plages/des bombardiers sur la Serbie/un vent de mort au Kosovo» [153]). À ces perspectives concrètes et fort médiatiques, le poète préfère plutôt un point de vue très englobant, qui pourrait être celui de Dieu - ou du poète devenu Dieu et présent à tout le réel d'un seul coup :

\author{
la Terre baigne dans l'air \\ il y a du ciel partout \\ l'air tient tout entier dans la lumière du ciel (129).
}

Une métaphysique amusante vient alors interroger les certitudes sur lesquelles nous vivons : "Au commencement était le corps, et le corps était une âme, à muscles lents et à cuir chevelu.» (137) La fantaisie domine, avec ses astuces et ses questions inopinées: «À quoi songe un sexe d'homme au cœur d'un sexe de femme?» (154)

Les poèmes de Jacques Rancourt sont pleins de très jolis éclairs, qui reflètent la beauté des titres de ses recueils, Le pont verbal, La condition terrestre, La nuit des millepertuis, L'instant prodigue, La pluie des pluies... Un régal pour ce qui est du détail des textes, et une attente peut-être moins comblée pour ce qui touche l'essentiel du contenu.

Hélène Dorion, dont j'ai salué naguère la monumentale rétrospective poétique ${ }^{6}$, fait de plus en plus figure de poète marquante dans notre paysage littéraire. Les nombreux prix et honneurs qu'elle a reçus, au pays et ailleurs, témoignent de son rayonnement considérable. Tout en reconnaissant son incontestable talent, j'ai formulé déjà des réserves sur une inspiration qui me semble un peu trop dualiste ${ }^{7}$, et L'étreinte des $v^{v e n t s}{ }^{8}$, malgré le prix prestigieux qui lui est décerné, m'inspire aujourd'hui de semblables commentaires.

$$
++
$$

6 Hélène Dorion, Mondes fragiles, choses frêles. Poèmes 1983-2000, Montréal, l'Hexagone, coll. «Rétrospectives », 2006, 802 p. Voir mon compte rendu dans «Les mots-motifs d'Hélène Dorion, et deux auteurs d'un seul livre: Miron/Gaulin", Voix et Images, vol. XXXII, n 3, printemps-été 2007, p. 139-143. 7 Par exemple, dans une chronique au titre saugrenu, j'en conviens à genoux: "Fredons nerd», Voix et Images, vol. XXV, n 1 , automne 1999, p. 200-203. 8 Hélène Dorion, L'étreinte des vents, Montréal, Prix de la revue Études françaises, Presses de l'Université de Montréal, 2009, 144 p. 
Il s'agit d'un essai dont le discours concilie avec bonheur poésie et philosophie, tout en portant en filigrane le récit d'une rupture amoureuse et d'une reconquête de la «joie de vivre» (63) - l'auteure ne répugne pas aux formules toutes faites. Hélène Dorion y reprend et approfondit les thèmes principaux de son œuvre, à travers des motifs insistants. L'idée de lien, particulièrement développée dès le début, en rapport avec la théorie du physicien David Böhm ( «l'univers serait une structure infinie d'ondes dans laquelle tout est lié à tout, et où les contraires [...] ne sont que des manifestations différentes d'une même réalité» [12]), et récurrente tout au long du texte, exprime la nécessaire rencontre de l'Autre qui fonde la vie, et qui fonde du même coup l'Amour ("avec un grand A», est-il précisé [18]). On retrouve là une morale, ou en tout cas une éthique, aux puissants relents de catholicisme, encore que «l'Aiméee» (sic), objet du sentiment, ne soit réductible à aucune orthodoxie religieuse.

Or, les liens qu'on multiplie avec le monde, dans une perspective amoureuse, sont facilement brisés par l'existence, et l'on expérimente aussitôt le chaos, la rupture, la solitude. Dès lors, «plus de lumière pour définir l'ombre, plus d'ombre pour têmoigner de la lumière ( (34). Les contraires semblent se dissoudre dans un vague immense, un «brouillard» (34), un «magma» $(31,34)$, et pourtant, ce vague joue, par rapport au défini, le même rôle que l'ombre par rapport au jour. Bref, on ne sort pas de la dualité du Bien et du Mal (ou, en termes plus laïques, de l'être et du néant). Que le vague résulte immanquablement de la confrontation des contraires montre bien que la médiation, que toute dialectique sont impossibles. Les contraires évoluent soit dans le sens de leur annulation limbique, soit dans l'exultation extrémiste, lumière et ombre devenant alors des infinis substituables l'un à l'autre. Pas étonnant, dès lors, que la rencontre du monde soit la rencontre de soi : «Émerveillée, je regarde cet horizon vaste et plein, à la fois familier et inconnu, et je sais que c'est à l'intérieur de moi que se posent ainsi mes yeux.» (64) L'autre, c'est moi.

Une telle métaphysique mène à des considérations compliquées. Elles ont le mérite d'éclairer la poésie de l'auteure, toujours propulsée de thème en antithèse, de faille (27) ou de brisure (50) en fragiles éternités (478). Chez Hélène Dorion, tout a la splendeur bleutée de l'infini et tout, presque simultanément, vire au magma inqualifiable, en raison d'une essentielle fragilité du monde.

Mais, comme chaque aspect du monde contient son contraire, le salut est constamment à portée de la main. La perte aussi. Une sorte d'arbitraire thématique en résulte peut-être. 